\title{
Caracterización de la crianza de cuyes en tres provincias de la Región Cajamarca, Perú
}

\author{
Characterization of the breeding of guinea pigs in three provinces of the Cajamarca \\ Region, Peru
Pedro Ortiz-Oblitas ${ }^{1,4}$, Amarante Florián-Alcántara ${ }^{2}$, Judith Estela-Manrique ${ }^{2}$, Marco Rivera-Jacinto ${ }^{3}$, Cristian Hobán-Vergara ${ }^{1}$, César Murga-Moreno ${ }^{1}$

\section{RESUMEN}

\begin{abstract}
El presente estudio tuvo como objetivo la caracterización de la crianza de cuyes en tres provincias de la Región Cajamarca, Perú. Se aplicó una encuesta estructurada a 1231 productores (401 de Cajamarca, 360 de San Marcos y 470 de Cajabamba). El sistema de producción predominante es el familiar-comercial (67\% de productores). La raza Perú es la preferida por los productores, utilizando mayormente el sistema de crianza en pozas. La mayoría de los productores posee menos de 49 cuyes con un área de terreno entre 0.5 a 5 ha., siendo la alfalfa (Medicago sativa) el forraje más utilizado en la alimentación de los cuyes. Más del $80 \%$ de productores no reciben asistencia técnica y más del $87 \%$ no forman parte de alguna asociación de criadores. En la caracterización sanitaria, entre 1 y 5 cuyes mueren por semana y por productor, especialmente en la clase lactantes. La enfermedad más reportada es la salmonelosis, la cual es identificada por las alteraciones en hígado.
\end{abstract}

Palabras clave: cuyes, Cajamarca, San Marcos, Cajabamba, producción, sanidad

\footnotetext{
${ }^{1}$ Laboratorio de Inmunología, Facultad de Ciencias Veterinarias, Universidad Nacional de Cajamarca, Perú

${ }^{2}$ Estación Experimental Baños del Inca, Instituto Nacional de Innovación Agraria, Cajamarca, Perú

${ }^{3}$ Laboratorio de Microbiología, Facultad de Ciencias de la Salud, Universidad Nacional de Cajamarca, Perú

${ }^{4}$ E-mail: portiz@unc.edu.pe
}

Fuente de financiamiento: Proyecto 004-PI, Programa Nacional de Innovación Agraria

Recibido: 8 de julio de 2020

Aceptado para publicación: 14 de enero de 2021

Publicado: 24 de abril de 2021 
The present study aimed to characterize the guinea pig breeding system in three provinces of the Cajamarca Region, Peru. A structured survey was applied to 1231 producers (401 from Cajamarca, 360 from San Marcos and 470 from Cajabamba). The predominant production system is family-commercial (67\% of producers). The Peru breed is preferred by producers, rearing the animals in pools. Most of the producers have less than 49 guinea pigs in a 0.5 to 5 ha land area. Alfalfa (Medicago sativa) being the forage most used in the feeding of guinea pigs. More than $80 \%$ of producers do not receive technical assistance and more than $87 \%$ are not part of a breeder association. In the sanitary characterization, 1 to 5 guinea pigs die per week and per producer, especially in the lactating class. The most reported disease is salmonellosis, which is identified by alterations in the liver.

Key words: guinea pigs, Cajamarca, San Marcos, Cajabamba, production, health

\section{INTRODUCCIÓN}

La seguridad alimenticia se define como el acceso físico, social y económico a suficientes cantidades de alimento seguro y nutritivo para cubrir las necesidades dietéticas de una vida saludable. Los sistemas de producción animal tienen que ser diseñados para abastecer un incremento cada vez más demandante de proteína de origen animal para el consumo humano (FAO et al., 2012). La crianza del cuy en el Perú ha tenido un desarrollo notable, llegando a ser una fuente de excelente aporte nutricional para el consumidor, particularmente por su alto contenido proteico (Bolton, 1979; Higaona et al., 2008). Cabe indicar que la carne de cuy ha sido alimento esencial para las comunidades altoandinas desde tiempos precolombinos, especialmente para los sectores de menores recursos económicos (Rosenfeld, 2008).

El cuy fue domesticado hace unos 3000-6000 años en los valles interandinos de Sudamérica (Gade, 1967; Lanning, 1967; Kyle, 1994), habiéndose convertido en una fuente importante de ingresos económicos para los productores a través de la venta de animales para consumo de carne y excedentes para fines reproductivos (FONCODES, 2014; Ngoula et al., 2017). El Perú es uno de los países con mayor producción de cuyes a nivel mundial (Chauca, 1997; Chauca et al., 2008; INEI, 2012), cuya crianza se lleva a cabo principalmente bajo el sistema familiar, seguido del familiar-comercial y el comercial (Chauca et al., 1994).

Cajamarca es una región eminentemente productora de cuyes, considerándose actualmente como la primera región productora del Perú, con una población de más de dos millones de ejemplares; seguida de la Región Cusco con 1.7 millones y la Región Ancash con 1.6 millones (INEI, 2012). Si bien Cajamarca es una de las regiones más importantes en la crianza de cuyes, se dispone de escasa información sobre la caracterización productiva relacionada a los factores de manejo y aspectos de sanidad en los sistemas productivos de la zona; es así que, el objetivo del presente estudio fue conocer los parámetros productivos más relevantes en la crianza de cuyes en tres de las principales provincias productoras de la Región Cajamarca. 


\section{Materiales y Métodos}

El trabajo se llevó a cabo entre julio y septiembre de 2016 en tres de las principales provincias productoras de la Región Cajamarca: Cajamarca, San Marcos y Cajabamba, ubicadas en la parte sur oeste de la Región.

La Región Cajamarca está situada en la zona nor-andina, presentando zonas de sierra y selva. Es la quinta Región más poblada del país con una población de 1341012 habitantes (INEI, 2017a). El sector agropecuario es la actividad económica más importante y el potencial económico se sustenta en la riqueza de sus valles interandinos y en la abundancia de recursos naturales (INEI, 2017b). La provincia de Cajamarca está situada a una altitud de $2720 \mathrm{msnm}$, tiene 348433 habitantes y cuenta con 12 distritos. La provincia de San Marcos se encuentra a $63 \mathrm{~km}$ de la ciudad de Cajamarca (INEI, 2001), a 2257 msnm, cuenta con una población de 54628 habitantes (INEI, 2017a), está conformada por siete distritos y comúnmente se le denomina el «Granero del Norte» por su amplia riqueza agrícola. La provincia de Cajabamba presenta una población de 50275 habitantes, está situada a 2648 msnm y cuenta con cuatro distritos, siendo sus principales actividades económicas la producción agropecuaria, extracción minera, forestal y servicios turísticos (INEI, 2017b).

Para el levantamiento de la información se elaboró y aplicó una encuesta estructurada, a través de una entrevista personal, que constituyó la herramienta principal para la interacción del encuestador con los productores. La encuesta fue validada mediante estudio piloto y opinión de expertos. El instrumento estuvo conformado por 40 preguntas cerradas con 2 a 8 opciones para marcar, distribuidas en cuatro temas de interés: datos generales del propietario, información de la actividad agropecuaria, información de la crianza de cuyes y estado sanitario de los cuyes. El tiempo promedio que tomó la encuesta fue de 25 minutos.
La encuesta fue aplicada por tres encuestadores, uno por zona, que fueron capacitados en dos sesiones de trabajo por el equipo de investigación. Los encuestadores se presentaron en forma independiente, mediante una carta de presentación otorgada por el director del proyecto. Los encuestados manifestaron su aceptación de participar en el estudio firmando un consentimiento informado. La información correspondiente a la identificación de los encuestados fue codificada y mantenida, bajo custodia, por el investigador responsable. La base de datos construida para su análisis no contiene información personal de los encuestados. Las encuestas se aplicaron en una sola visita a 1231 productores de cuyes de las tres provincias en estudio. El número de encuestas por provincia fue de 401 para Cajamarca, 360 para San Marcos y 470 para Cajabamba. La encuesta tuvo un nivel de rechazo del 1\% aproximadamente. El estudio estuvo basado en la metodología para la aplicación del enfoque de sistemas en América Latina (Escobar, 1995). Posteriormente, los datos fueron analizados mediante análisis descriptivo.

\section{Resultados y Discusión}

La distribución de los productores encuestados por provincia y distrito se presentan en el Cuadro 1. La mayoría de los productores de la zona en estudio utilizan el sistema de crianza familiar comercial (53.9, 73.9 y $74.0 \%$ de productores de Cajamarca, San Marcos y Cajabamba respectivamente), sistema que es el más empleado en el país (Chauca et al., 2008), mientras que entre 0.5 y $2.2 \%$ de productores se dedican a la crianza de cuyes bajo un sistema comercial (Cuadro 2), con una población que supera a los 500 animales por productor (Chauca et al., 2008). Esto es importante a tener en consideración, ya que Cajamarca es una de las regiones con mayor nivel de pobreza en el país (INEI, 2017a) y la crianza de cuyes podría convertirse en una actividad generadora de recursos y de fuente de trabajo sostenible 
Cuadro 1. Productores de cuyes encuestados en tres provincias productoras de la Región Cajamarca, Perú (2016)

\begin{tabular}{|c|c|c|c|}
\hline Provincia & Distrito & $\begin{array}{l}\text { Productores } \\
\text { (n) }\end{array}$ & $\begin{array}{l}\text { Cuyes } \\
\text { (miles) }\end{array}$ \\
\hline \multirow[t]{7}{*}{ Cajamarca } & Cajamarca & 55 & 4,420 \\
\hline & Baños del Inca & 49 & 4,016 \\
\hline & Jesús & 140 & 14,544 \\
\hline & LLacanora & 55 & 4,118 \\
\hline & Namora & 3 & 560 \\
\hline & Matara & 97 & 9,999 \\
\hline & San Juan & 2 & 138 \\
\hline \multicolumn{2}{|c|}{ Sub total $(n=7)$} & 401 & 37,795 \\
\hline \multirow{5}{*}{ San Marcos } & Chancay & 40 & 1,570 \\
\hline & Eduardo Villanueva & 74 & 19,432 \\
\hline & Ichocán & 16 & 870 \\
\hline & José Manuel Quiroz & 22 & 1,070 \\
\hline & Pedro Gálvez & 208 & 38,844 \\
\hline \multicolumn{2}{|c|}{ Sub total $(n=5)$} & 360 & 61,786 \\
\hline \multirow{3}{*}{ Cajabamba } & Cachachi & 53 & 21,340 \\
\hline & Cajabamba & 70 & 12,728 \\
\hline & Condebamba & 347 & 70,757 \\
\hline \multicolumn{2}{|c|}{ Sub total $(n=3)$} & 470 & 104,825 \\
\hline Total & 15 & 1,231 & 204,406 \\
\hline
\end{tabular}

para muchas familias. El tipo de crianza ha evolucionado en los últimos años, desde la crianza más tradicional en pozas hasta la crianza en jaulas o baterías, mayormente usada por empresas comerciales (FONCODES, 2014). En el presente estudio, la crianza en pozas (Huamán et al., 2019) fue la más usada en las tres provincias en estudio (Cuadro 2).

La mayor parte de los productores mostró preferencia por cuyes de la raza Perú (61.6-94.2\%), debido a su alta productividad, precocidad y excelente calidad de carne (Chauca et al., 2004; Collado, 2016) (Cuadro 2). La mayoría de los productores tienen menos de 50 animales, utilizados para el autoconsumo y la venta local, lo cual convierte a la crianza de cuyes en su única o principal fuente de ingreso familiar (Cuadro 2). Se conoce que, solo en el distrito de Cajabamba se comercializan entre 5 a 10 mil cuyes por mes (Municipalidad Provincial de Cajabamba, 2007).

En el Cuadro 3 se puede apreciar, asimismo, que la gran mayoría de productores posee entre 0.5 a 5 ha de terreno, área que se distribuye principalmente en las instalaciones para la crianza de cuyes, así como para la siembra de los forrajes utilizados, siendo la alfalfa (Medicago sativa) mayoritariamente usada como alimento de los cuyes. De otra 
Cuadro 2. Caracterización del manejo de los productores de cuyes de tres provincias de la Región Cajamarca, Perú, 2016 (Parte 1)

\begin{tabular}{|c|c|c|c|c|c|c|}
\hline \multirow{2}{*}{ Característica de manejo } & \multicolumn{2}{|c|}{ Cajamarca } & \multicolumn{2}{|c|}{ San Marcos } & \multicolumn{2}{|c|}{ Cajabamba } \\
\hline & $\mathrm{n}=401$ & $\%$ & $\mathrm{n}=360$ & $\%$ & $\mathrm{n}=470$ & $\%$ \\
\hline \multicolumn{7}{|l|}{ Sistema de producción } \\
\hline Tradicional & 176 & 43.9 & 74 & 20.6 & 105 & 22.3 \\
\hline Familiar-comercial & 216 & 53.9 & 266 & 73.9 & 348 & 74.0 \\
\hline Comercial & 2 & 0.5 & 8 & 2.2 & 6 & 1.3 \\
\hline No sabe/ no precisa & 7 & 1.7 & 12 & 3.3 & 11 & 2.3 \\
\hline \multicolumn{7}{|l|}{ Sistema de crianza } \\
\hline Pozas & 231 & 57.6 & 244 & 67.8 & 182 & 38.7 \\
\hline Jaulas & 73 & 18.2 & 28 & 7.8 & 108 & 23 \\
\hline Pozas más jaulas & 64 & 16.0 & 62 & 17.2 & 152 & 32.3 \\
\hline Baterías & 1 & 0.2 & 24 & 6.7 & 3 & 0.6 \\
\hline Otros & 32 & 8.0 & 2 & 0.6 & 25 & 5.3 \\
\hline \multicolumn{7}{|l|}{ Raza de cuyes que cría } \\
\hline Perú & 247 & 61.6 & 339 & 94.2 & 409 & 87.0 \\
\hline Andina & 8 & 2.0 & 0 & 0 & 2 & 0.4 \\
\hline Otras & 6 & 1.5 & 0 & 0 & 2 & 0.4 \\
\hline Más de una raza & 140 & 34.9 & 21 & 5.8 & 57 & 12.1 \\
\hline \multicolumn{7}{|l|}{ Población total de animales } \\
\hline 1 a 49 & 364 & 90.8 & 207 & 57.5 & 290 & 61.7 \\
\hline 50 a 100 & 22 & 5.5 & 85 & 23.6 & 116 & 24.7 \\
\hline 101 a 500 & 15 & 3.7 & 63 & 17.5 & 58 & 12.3 \\
\hline 501 a más & 0 & 0 & 5 & 1.4 & 6 & 1.3 \\
\hline \multicolumn{7}{|l|}{ Venta mensual de animales } \\
\hline 10 a 49 & 386 & 96.3 & 293 & 81.4 & 420 & 89.4 \\
\hline 50 a 99 & 11 & 2.7 & 39 & 10.8 & 26 & 5.5 \\
\hline 100 a 150 & 3 & 0.7 & 20 & 5.6 & 13 & 2.8 \\
\hline Más de 151 & 1 & 0.2 & 8 & 2.2 & 11 & 2.3 \\
\hline
\end{tabular}

parte, entre el 81.4 y $87.4 \%$ de los productores encuestados no reciben asistencia técnica alguna, ni por parte del Estado ni de organizaciones particulares; y más del $87 \%$ de los encuestados no se encuentra asociado a algún tipo de agrupación de productores (Cua- dro 3). Este resultado llama la atención, ya que Cajamarca es una de las principales regiones productoras de cuyes (INEI, 2012) del país, lo que requeriría una mayor atención por parte del Estado o de organizaciones privadas que brindan apoyo a este tipo de crianza. 
Cuadro 3. Caracterización del manejo de los productores de cuyes de tres provincias de la Región Cajamarca, Perú, 2016 (Parte 2)

\begin{tabular}{|c|c|c|c|c|c|c|}
\hline \multirow{2}{*}{ Característica de manejo } & \multicolumn{2}{|c|}{ Cajamarca } & \multicolumn{2}{|c|}{ San Marcos } & \multicolumn{2}{|c|}{ Cajabamba } \\
\hline & $\mathrm{n}=401$ & $\%$ & $n=360$ & $\%$ & $\mathrm{n}=470$ & $\%$ \\
\hline \multicolumn{7}{|l|}{$\begin{array}{l}\text { Área de terreno para } \\
\text { forraje (ha) }\end{array}$} \\
\hline 0.5 a 5 & 392 & 97.8 & 359 & 99.7 & 459 & 97.7 \\
\hline Más de 5 & 0 & 0 & 0 & & 4 & 0.9 \\
\hline Más de 10 & 0 & 0 & 0 & 0 & 0 & 0 \\
\hline Menos de 0.5 & 0 & 0 & 0 & 0 & 6 & 1.3 \\
\hline No tiene/no precisa & 9 & 2.2 & 1 & 0.3 & 1 & 0.2 \\
\hline \multicolumn{7}{|l|}{ Forrajes que cultiva } \\
\hline Alfalfa & 192 & 47.9 & 252 & 70 & 451 & 96 \\
\hline $\begin{array}{l}\text { Alfalfa }+ \text { otros } \\
\text { forrajes }\end{array}$ & 174 & 43.4 & 107 & 29.7 & 13 & 2.8 \\
\hline Otros forrajes & 35 & 8.7 & 0 & 0 & 6 & 1.3 \\
\hline No cultiva & 0 & 0 & 1 & 0.3 & 0 & 0 \\
\hline \multicolumn{7}{|l|}{ Recibe asistencia técnica } \\
\hline Sí & 59 & 14.7 & 66 & 18.3 & 52 & 11.1 \\
\hline No & 342 & 85.3 & 293 & 81.4 & 411 & 87.4 \\
\hline No informa & 0 & 0 & 1 & 0.3 & 7 & 1.5 \\
\hline \multicolumn{7}{|c|}{ Pertenece a alguna asociación } \\
\hline Sí & 47 & 11.7 & 18 & 5 & 54 & 11.5 \\
\hline No & 354 & 88.3 & 342 & 95 & 412 & 87.7 \\
\hline
\end{tabular}

La sanidad animal es una herramienta indispensable en la producción animal, no solo porque contribuye a la producción de alimentos de calidad sino porque, a través de esta, se provee de ingresos a pequeños productores de los países en desarrollo (FAO, 2019). Diversas enfermedades afectan la productividad en la crianza de cuyes (Guerra, 2009; Huamán et al., 2019). En el presente estudio se encontró que la gran mayoría de productores refieren que se les muere entre 1 y 5 cuyes por semana, especialmente en la clase lactantes $(49.4 \%$ en Cajamarca, $40.0 \%$ en
San Marcos y 27.2\% en Cajabamba) (Cuadro 4).

La salmonelosis, enfermedad comúnmente denominada «peste» por los lugareños (Aguilar et al., 2011), fue referida como uno de los principales problemas sanitarios que se presentan en los criadores de cuyes, con valores porcentuales que fluctuaron entre 34.9 y $69.7 \%$ en las tres provincias estudiadas (Cuadro 4). El agente causal de la salmonelosis en cuyes ha sido aislado por diversos investigadores (Layme et al., 2011; 
Cuadro 4. Caracterización de la sanidad de los productores de cuyes de tres provincias de la Región Cajamarca, Perú, 2016

\begin{tabular}{lcccccc}
\hline \multirow{2}{*}{ Característica de sanidad } & \multicolumn{2}{c}{ Cajamarca } & \multicolumn{2}{c}{ San Marcos } & \multicolumn{2}{c}{ Cajabamba } \\
\cline { 2 - 7 } & $\mathrm{n}=401$ & $\%$ & $\mathrm{n}=360$ & $\%$ & $\mathrm{n}=470$ & $\%$ \\
\hline Cuyes que mueren por semana $(\mathrm{n})$ & & & & & \\
1 a 5 & 382 & 95.26 & 310 & 86.1 & 455 & 96.8 \\
6 a 10 & 8 & 2 & 8 & 2.2 & 5 & 1.1 \\
11 a 20 & 0 & 0 & 0 & 0 & 2 & 0.4 \\
Más de 20 & & 0 & 5 & 1.4 & 0 & 0 \\
No se le mueren & 11 & 2.74 & 37 & 10.3 & 8 & 1.7 \\
Clase de cuyes que mueren & & & & & & \\
Reproductores & 79 & 19.7 & 44 & 12.2 & 27 & 5.7 \\
Recría & 67 & 16.7 & 3 & 0.8 & 20 & 4.3 \\
Lactantes & 198 & 49.4 & 144 & 40.0 & 128 & 27.2 \\
Más de una opción & 53 & 13.2 & 131 & 36.4 & 295 & 62.8 \\
No sabe & 4 & 1 & 38 & 10.56 & 0 & 0 \\
¿Qué hace con los cuyes enfermos? & & & & & \\
Aísla & 185 & 46.1 & 343 & 95.3 & 269 & 57.2 \\
Sacrifica y sepulta & 60 & 15.0 & 3 & 0.8 & 18 & 3.8 \\
Trata con medicamento & 40 & 10.0 & 2 & 0.6 & 3 & 0.6 \\
Más de una opción & 64 & 16.0 & 5 & 1.4 & 148 & 31.5 \\
No toma medidas & 52 & 13.0 & 7 & 1.9 & 32 & 6.8 \\
¿Qué hace con los cuyes que mueren? & & & & & \\
Entierra & 251 & 62.6 & 323 & 89.7 & 231 & 49.2 \\
Pozo séptico & 39 & 9.7 & 5 & 1.4 & 45 & 9.6 \\
Arroja a la chacra/perros & 57 & 14.2 & 7 & 1.94 & 79 & 16.8 \\
Más de una opción & 41 & 10.2 & 4 & 1.1 & 108 & 23.0 \\
Ninguna opción & 13 & 3.2 & 21 & 5.8 & 7 & 1.5 \\
¿Qué observa a la necropsia? & & & & & & \\
Problemas hepáticos & 189 & 47.1 & 326 & 90.5 & 90 & 19.2 \\
Bazo agrandado & 10 & 2.5 & 0 & 0 & 7 & 1.5 \\
Intestino sanguinolento & 4 & 1 & 1 & 0.23 & 1 & 0.2 \\
Más de una opción & 71 & 17.7 & 18 & 5 & 303 & 64.5 \\
Otras opciones & 26 & 6.5 & 0 & 0 & 43 & 9.2 \\
No los abre & 101 & 25.2 & 15 & 4.2 & 26 & 5.5 \\
Principal problema observado & & & & & & \\
Salmonelosis & 140 & 34.9 & 251 & 69.7 & 215 & 45.7 \\
Neumonía & 4 & 1 & 13 & 3.6 & 6 & 1.3 \\
Linfadenitis & 5.5 & 4 & 1.1 & 7 & 1.5 \\
Parásitos & 10.5 & 87 & 24.2 & 3 & 0.6 \\
Otras opciones & 46.9 & 0 & 0 & 239 & 50.8 \\
No sabe & 1.2 & 5 & 1.4 & 0 & 0 \\
\hline
\end{tabular}


Ortega et al., 2015; Chero et al., 2017; Chuquizuta y Morales, 2017), lo cual, aparte del problema sanitario que causa en los animales, constituye un mecanismo de contaminación y peligro desde el punto de vista de salud pública (Humphrey y Jørgensen, 2006). Los productores utilizan diversos antibióticos para el tratamiento (Alegría, 2008; Jara, 2014), con la consecuente aparición de los fenómenos de resistencia antimicrobiana tanto en animales como en el hombre (Aarestrup y Wegener, 1999; Wendlandt et al., 2015; Scott et al., 2018).

Finalmente, es importante notar que, los productores, por las lesiones que observan a nivel de hígado al momento de realizar la necropsia de los animales (Cuadro 4) reconocen a la salmonelosis como la enfermedad causante de la muerte de sus animales (Parra et al., 2002; Layme et al., 2011).

\section{Conclusiones}

- La crianza de cuyes en la Región Cajamarca se realiza mayormente bajo el sistema de producción familiar-comercial, con poca asistencia técnica, con crianza predominantemente en pozas y alimentación basada en forraje verde (alfalfa).

- La enfermedad que más afecta a los cuyes es la salmonelosis, con lesiones principalmente a nivel de hígado.

\section{Agradecimiento}

Los autores expresan su agradecimiento al Programa Nacional de Innovación Agraria, Proyecto 004-PI, Programa Nacional de Innovación Agraria, por el financiamiento brindado para el desarrollo del presente estudio.

\section{Literatura Citada}

1. Aarestrup F, Wegener H. 1999. The effects of antibiotic usage in food animals on the development of antimicrobial resistance of importance for humans in Campylobacter and Escherichia coli. Microbes Infect 8: 639-644. doi: 10.1016/ s1286-4579(99)80064-1

2. Aguilar V, Bustamante J, Bazán V, Falcón N. 2011. Diagnóstico situacional de la crianza de cuyes en el distrito de Santa Cruz, Cajamarca. Rev Inv Vet Perú 22: 9-14.

3. Alegría C. 2008. Efecto de la norfloxacina en el tratamiento de Salmonella spp en cuyes. Tesis de Ingeniero Zootecnista. Tingo María, Perú: Univ. Nacional Agraria de la Selva. 53 p.

4. Bolton R. 1979. Guinea pigs. Protein and ritual. Ethnology 18: 229-252.

5. Chauca L. 1997. Producción de cuyes (Cavia porcellus). FAO, Roma: Organización de las Naciones Unidas para la Agricultura y la Alimentación. 93 p.

6. Chauca L, Herrera L, Caldas $N$, 2004. Evaluación del crecimiento de cuyes raza Perú alimentados con raciones con diferente densidad nutricional. En: XXVII Reunión de la Asociación Peruana de Producción Animal.

7. Chauca L, Zaldivar M, Muscari J, Higaona R, Gamarra J, Florian A. 1994. Proyecto Sistemas de Producción de Cuyes. Informe Técnico. Tomo I. [Internet]. Disponible en: http:// www.inia.gob.pe/documentos/

8. Chauca, L, Higaonna, R, Muscari, J. 2008. Investigaciones en cuyes. Informe Técnico. Tomo II. [Internet]. Disponible en: http://www.inia.gob.pe/documentos/

9. Chero A, Rosadio R, Marcelo G, Díaz G, Jiménez R, Castro Y, Maturrano L. 2017. Identificación molecular de Salmonella Typhimurium en cuyes al primer parto mediante la técnica de PCR múltiple. Rev Inv Vet Perú 28: 679-686. doi: 10.15381/rivep.v28i3.13288

10. Chuquizuta C, Morales S. 2017. Identificación de agentes bacterianos aislados de gazapos muertos de cuyes en una granja de crianza intensiva en Lima, Perú. REDVET 18: 13 p. 
11. Collado K. 2016. Ganancia de peso en cuyes machos (Cavia porcellus), post destete de la raza Perú, con tres tipos de alimento: balanceado, mixta, testigo (alfalfa) en Abancay. Tesis de Ingeniero Agrónomo. Abancay, Perú: Univ. Tecnológica de los Andes. $92 \mathrm{p}$.

12. Escobar G 1995. Desarrollo metodológico para la aplicación del enfoque de sistemas en América Latina. En: Verdegue J, Ramirez E (eds). Investigación con enfoque de sistemas en la agricultura y el desarrollo rural. Santiago de Chile: RIMISP. 348 p.

13. [FAO] Organización de las Naciones Unidas para la Agricultura y la Alimentación. 2019. Sanidad animal. Rome: FAO. [Internet]. Disponible en: http://www.fao.org/animal-health/es/

14. FAO, WFP, IFAD. 2012. The state of food insecurity in the world [Internet]. Available in: http://www.fao.org/3/ai3027e.pdf

15. [FONCODES] Fondo de Cooperación para el Desarrollo Social. 2014. Crianza de cuyes: Manual Técnico N. ${ }^{\circ} 4$. Lima, Perú: Ministerio de Desarrollo e Inclusión Social. $45 \mathrm{p}$.

16. Gade D. 1967. The guinea pig in Andean folk culture. Am Geogr Soc 57: 213-224.

17. Guerra C. 2009. Manual técnico de crianza de cuyes. Cajamarca, Perú: CEDEPAS. 25 p.

18. Higaona OR, Muscari GJ, Chauca FL, Astete F. 2008. Composición química de la carne de cuy. En: XXXI Reunión Anual de la Asociación Peruana de Producción Animal. Lima, Perú.

19. Huamán M, Killerby M, Chauca L. 2019. Manual de bioseguridad y sanidad en cuyes. Lima, Perú: Instituto Nacional de Innovación Agraria - INIA. 86 p. [Internet]. Disponible en: http:// repositorio.inia.gob.pe/handle/inia/936

20. Humphrey T, Jorgensen F. 2006. Pathogens on meat and infection in animals - Establishing a relationship using Campylobacter and Salmonella as examples. Meat Sci 74: 89-97. doi: 10.1016/j.meatsci.2006.04.026
21. [INEI] Instituto Nacional de Estadística e Informática. 2001. Conociendo Cajamarca, Lima, Perú. [Internet]. Disponible en: https://www.inei.gob.pe/media/MenuRecursivo/publicaciones_digitales/Est/Lib0409/Libro.pdf INEI

22. [INEI] Instituto Nacional de Estadística e Informática. 2012. IV Censo Nacional Agropecuario 2012. Lima, Perú. [Internet]. Disponible en: http:// censos.inei.gob.pe/Cenagro/redatam/

23. [INEI] Instituto Nacional de Estadística e Informática. 2017a. Censos Nacionales 2017: XII de Población, VII de Vivienda y III de Comunidades Indígenas. Lima, Perú. [Internet]. Disponible en: http://censo2017.inei.gob.pe/

24. [INEI] Instituto Nacional de Estadística e Informática. 2017b. Cajamarca Compendio Estadístico. Cajamarca, Perú. [Internet]. Disponible en: https:// w w w i n e i.gob.pe/media/ MenuRecursivo/publicaciones_digitales/ Est/Lib1492/libro.pdf

25. Jara E. 2014. Evaluación de cinco fármacos para el tratamiento de cuyes (Cavia porcellus) infectados experimentalmente con Salmonella (S. tiphymurium), en la irrigación Majes, provincia de Caylloma, Departamento de Arequipa, 2013. Tesis de Médico Veterinario y Zootecnista. Arequipa, Perú: Univ. Católica de Santa María. 104 p.

26. Kyle R. 1994. New species for meat production. J Agric Sci 123: 1-8. doi: 10.1017/S0021859600067708

27. Lanning E. 1967. Peru before the Incas. UK: Prentice Hall $216 \mathrm{p}$.

28. Layme A, Perales $R$, Chavera A, Gavidia C, Calle S. 2011. Lesiones anatomopatológicas en cuyes (Cavia porcellus) con diagnóstico bacteriológico de Salmonella sp. Rev Inv Vet Perú 22:369-376.

29. Municipalidad Provincial de Cajabamba. 2007. Plan de Desarrollo Concertado de la Provincia Cajabamba al 2017. [Internet]. Disponible en: https:/ /www.peru.gob.pe/docs/PLANES/ 
11882/PLAN_11882_Plan_Provincial_de_Desarrollo_Concertado_2017_2011.pdf

30. Ngoula F, Guemdjo Tekam M, Kenfack A, Tadondjou Tchingo C, Nouboudem S, Tsafack B, Teguia A, et al. 2017. Effects of heat stress on some reproductive parameters of male cavie (Cavia porcellus) and mitigation strategies using guava (Psidium guajava) leaves essential oil. J Therm Biol 64: 67-72. doi: 10.1016/j.jtherbio.2017.01.001

31. Ortega G, Jiménez R, Ara M, Morales $S$. 2015. La salmonelosis como factor de riesgo de mortinatalidad en cuyes. Rev Inv Vet Perú 26: 676-681. doi: 10.15381/rivep.v26i4.11203

32. Parra M, Durango J, Máttar S. 2002. Microbiología, patogénesis, epidemiología, clínica y diagnóstico de las infecciones producidas por Salmonella. Rev MVZCórdoba 7: 187-200. doi: 10.21897/ rmvZ.521
33. Rosenfeld SA. 2008. Delicious guinea pigs: seasonality studies and the use of fat in the pre-Columbian Andean diet. Quat Int 180: 127-134. doi: 10.1016/ j.quaint.2007.08.011

34. Scott A, Beller E, Glasziou P, Clark J, Ranakusuma R, Byambasuren $O$, Bakhit M, Page S, et al. 2018. Is antimicrobial administration to food animals a direct threat to human health? A rapid systematic review. Int $\mathrm{J}$ Antimicrob Agents 3: 316-323. doi: / 10.1016/j.ijantimicag.2018.04.005

35. Wendlandt S, Shen J, Kadlec K, Wang Y, Li B, Zhang W, Feßler AT, et al. 2015. Multidrug resistance genes in staphylococci from animals that confer resistance to critically and highly important antimicrobial agents in human medicine. Trends Microbiol 23: 44-54. doi: 10.1016/j.tim.2014.10.002 